\title{
Strong-Weak Nonlinear Bilevel Problems: Existence of Solutions in a Sequential Setting
}

\author{
Abdelmalek Aboussoror ${ }^{1}$ - Samir Adly ${ }^{2}$. \\ Fatima Ezzahra Saissi ${ }^{1,2}$
}

\begin{abstract}
The paper deals with a strong-weak nonlinear bilevel problem which generalizes the well-known weak and strong ones. In general, the study of the existence of solutions to such a problem is a difficult task. So that, for a strong-weak nonlinear bilevel problem, we first give a regularization based on the use of strict $\epsilon$-solutions of the lower level problem. Then, via this regularization and under sufficient conditions, we show that the problem admits at least one solution. The obtained result is an extension and an improvement of some recent results appeared recently in the literature for both weak nonlinear bilevel programming problems and linear finite dimensional case.
\end{abstract}

Keywords Bilevel optimization - Convergence of multifunctions · Marginal functions · Variational-convergence $\cdot$ Convex analysis

Mathematics Subject Classification (2010) Primary: 91A65 - 90C30 - 90C31; Secondary: 46N10

Samir Adly

samir.adly@unilim.fr

Abdelmalek Aboussoror

aboussororabdel@hotmail.com

Fatima Ezzahra Saissi

fatima-ezzahra.saissi@etu.unilim.fr

1 Faculté Polydisciplinaire de Safi, Laboratoire LMC, Université Cadi Ayyad, B.P. 4162, Sidi Bouzid, Safi, Morocco

2 Université de Limoges, Laboratoire XLIM UMR-CNRS 6172, 123 Avenue Albert Thomas, 87060 Limoges Cedex, France 


\section{Introduction}

Let $X$ and $Y$ be two Hausdorff topological spaces, with $Y$ is first countable. We are concerned with the following bilevel optimization problem, called strong-weak Stackelberg problem

$$
(S): \quad \min _{x \in A} \inf _{y \in \mathcal{M}(x)} \sup _{z \in \mathcal{M}(x)} F(x, y, z)
$$

where $\mathcal{M}(x)$ is the solution set of the lower level problem

$$
\mathcal{P}(x): \quad \min _{z \in B} f(x, z)
$$

$F: X \times Y \times Y \rightarrow \mathbb{R}, f: X \times Y \rightarrow \mathbb{R}$ are functions, $A$ and $B$ are respectively two nonempty subsets of $X$ and $Y$. As it is well-known, bilevel optimization problems have applications in many areas; we cite for example, economics, transportation network design and engineering. The class of strong-weak bilevel problems has been first introduced in [1] (1995). Thereafter, other works have appeared on this class of problems; we cite for example $[11,13,14,18,23]$ and [26]. However, the majority of them deal with the linear finite dimensional case, for which algorithms and numerical results are given. For illustration of such a class of bilevel problems, let us give the following practical examples [1].

Example 1 (Stackelberg games) Let us consider a two-player game composed by a leader and a follower where the two players try to minimize their objective functions $\hat{F}$ and $\hat{f}$ respectively. Let $\hat{A}$ and $\hat{B}$ denote the sets of strategies of the leader and the follower respectively. The leader assumed to have all information about the follower, announces first a strategy $x \in \hat{A}$ and the follower reacts optimally by choosing a strategy $y(x) \in \hat{B}$. If the follower's solution set is not always reduced to a singleton (i.e., there exists $\hat{x} \in \hat{A}$ such that $\mathcal{M}(\hat{x})$ is not a singleton), then, two extreme cases are possible.

i) The pessimistic case : In this case the game is noncooperative. Then, the leader provides himself against the possible worst choice by the follower in $\mathcal{M}(x)$ by minimizing the marginal function

$$
\sup _{y \in \mathcal{M}(x)} \hat{F}(x, y) .
$$

ii) The optimistic case : In this case the follower is in full cooperation with the leader. So that, the leader minimizes the marginal function

$$
\inf _{y \in \mathcal{M}(x)} \hat{F}(x, y) .
$$

The two above cases lead to a weak and a strong Stackelberg problems respectively. Here, the terms "weak" and "strong" are taken in the sense of [10]. However, by taking into account the cooperation degree of the follower, these two cases can be obtained from the following general one. Then, if $\alpha \in[0,1]$ is the cooperation index between the two players, the leader minimizes the function

$$
\alpha \inf _{y \in \mathcal{M}(x)} \hat{F}(x, y)+(1-\alpha) \sup _{z \in \mathcal{M}(x)} \hat{F}(x, z) .
$$

Set $F_{\alpha}(x, y, z)=\alpha \hat{F}(x, y)+(1-\alpha) \hat{F}(x, z)$. Then, the leader's problem is formulated as

$$
\left(\hat{S}_{\alpha}\right) \quad \min _{x \in \hat{A}} \inf _{y \in \mathcal{M}(x)} \sup _{z \in \mathcal{M}(x)} F_{\alpha}(x, y, z)
$$


which is a strong-weak bilevel problem. Then, weak and strong bilevel problems are particular cases of problem $\left(\hat{S}_{\alpha}\right)$ which are respectively obtained for $\alpha=0$ and $\alpha=1$.

Example 2 (System of nondifferentiable convex inequalities) Let $f_{i}: \mathbb{R}^{n} \rightarrow \overline{\mathbb{R}}$, $i=1, \ldots, m$, be closed proper convex functions and consider the following system of inequalities

$$
f_{i}(x) \leq 0, i=1, \ldots, m .
$$

Set $C=\left\{x \in \mathbb{R}^{n} / f_{i}(x) \leq 0, i=1, \ldots m\right\}$ and $\theta(x)=\left(f_{1}^{+}(x), \ldots, f_{m}^{+}(x)\right)$, with $f_{i}^{+}(x)=$ $\max \left(0, f_{i}(x)\right), i=1, \ldots, m$. Auslender and Crouzeix in [7] have been interested by the best constant denoted by $k_{2, \infty}$ that satisfies

$$
d_{2}(x, C) \leq k_{2, \infty}\|\theta(x)\|_{\infty} \quad \text { i.e., } \quad k_{2, \infty}=\sup _{x \notin C} \frac{d_{2}(x, C)}{\|\theta(x)\|_{\infty}}
$$

where $d_{2}(x, C)=\min _{x \in C}\|x-y\|_{2},\|\cdot\|_{2}$ and $\|\cdot\|_{\infty}$ denote respectively the euclidean norm on $\mathbb{R}^{n}$ and the Tchebycheff norm on $\mathbb{R}^{m}$. Set $\hat{f}(x)=\max _{i=1, \ldots, m} f_{i}(x)$. Then, under appropriate assumptions, the authors show that the constant $k_{2, \infty}$ is given by

$$
k_{2, \infty}=\frac{1}{k_{2, \infty}^{*}} \quad \text { with } \quad k_{2, \infty}^{*}=\min _{x \in \operatorname{bd} C} \inf _{y \in \partial \hat{f}(x)} \sup _{z \in \partial \hat{f}(x)}\left\langle\frac{y}{\|y\|_{2}}, z\right\rangle
$$

where bd $C$ is the boundary of $C$ and $\partial \hat{f}(x)$ is the subdifferential of $\hat{f}$ at $x$ (see [25]). Define the functions

$$
\tilde{f}(x, z)=\hat{f}^{*}(z)-\langle x, z\rangle \quad \text { and } \quad \tilde{F}(x, y, z)=\left\langle\frac{y}{\|y\|_{2}}, z\right\rangle, y \neq 0
$$

where $\hat{f}^{*}$ is the conjugate function of $\hat{f}$. Set $\tilde{A}=\operatorname{bd} C$ and let $\mathcal{M}(x)$ denote the solution set of the problem

$$
\min _{z \in \mathbb{R}^{n}} \tilde{f}(x, z)
$$

Then, $\mathcal{M}(x)=\partial \hat{f}(x)$. Hence

$$
k_{2, \infty}^{*}=\min _{x \in \tilde{A}} \inf _{y \in \mathcal{M}(x)} \sup _{z \in \mathcal{M}(x)} \tilde{F}(x, y, z)
$$

which is the minimal value of a strong-weak Stackelberg problem.

Note that in the formulation above of problem $(S)$, when the function $F$ does not depend on $y$, we obtain a weak Stackelberg problem, and when $F$ does not depend on $z$, we obtain a strong Stackelberg problem. Therefore, strong-weak bilevel problems constitute a wide class of optimization problems which generalizes the class of weak and strong ones.

As it is well-known, weak nonlinear bilevel problems are difficult to solve from both theoretical and numerical aspects. Due to the generalization above, strong-weak bilevel problems present more difficulties. In fact, the problem $(S)$ consists to minimize the marginal function $u(x)=\inf _{y \in \mathcal{M}(x)} \sup _{z \in \mathcal{M}(x)} F(x, y, z)$ which has a more complicated expression than the marginal function considered in (1) for weak bilevel problems. In general, even if the data are convex and differentiable, the marginal function $u($.$) is neither$ convex nor differentiable. Furthermore, the multifunction $\mathcal{M}($.$) which represents the con-$ straints of $u($.) does not possess enough useful topological properties. This explains the difficulties encountered in particular in the study of the existence of solutions to such a class of problems. For instance, the bilevel problem $(S)$ may fail to have solutions even if the variables $x, y$ and $z$ are in compact sets and the objective functions $F$ and $f$ are continuous 
(see Example 3 below). This fact results principally from the lack of lower semicontinuity of the multifunction $\mathcal{M}($.). Then, let us summarize the following studies concerning this subject. The existence of solutions for weak and strong-weak bilevel problems in the linear finite dimensional case was established respectively in [4] and [26]. The studies are based on the use of linear duality and penalty methods. However, the nonlinear case presents more difficulties. For the finite dimensional case, sufficient conditions ensuring the existence of solutions to nonlinear weak bilevel programming problems are given in [3]. So that, it is interesting to consider the class of infinite dimensional nonlinear strong-weak bilevel problems. In this case, the obtained results shall also be applied to the infinite dimensional nonlinear weak bilevel problems by deleting the variable $y$ in the formulation of problem $(S)$ (that is when $F$ does not depend on $y$ ).

Let us consider the following example where the functions $F$ and $f$ are continuous and the variables $x, y$, and $z$ range over compact sets, but the problem $(S)$ has no solution.

Example 3 Let $X=\mathbb{R}^{2}, Y=\mathbb{R}, A=[0,1] \times[1,2], B=[0,1], F$ and $f$ be the functions defined respestively on $\mathbb{R}^{2} \times \mathbb{R} \times \mathbb{R}$ and $\mathbb{R}^{2} \times \mathbb{R}$ by

$$
F(x, y, z)=-x_{2} y z-\frac{1}{2} x_{1} x_{2}, f(x, z)=\frac{3}{2}\left(x_{2}-\frac{3}{2}\right) z+\frac{1}{4} x_{1}^{2}\left(x_{2}+1\right)^{2}
$$

with $x=\left(x_{1}, x_{2}\right)$. Then

$$
\mathcal{M}(x)= \begin{cases}\{1\} & \text { if } x_{2} \in\left[1, \frac{3}{2}[\right. \\ {[0,1]} & \text { if } x_{2}=\frac{3}{2} \\ \{0\} & \text { if } \left.\left.x_{2} \in\right] \frac{3}{2}, 2\right]\end{cases}
$$

which is a compact set for all $x \in A$. Set

$$
w(x, y)=\sup _{z \in \mathcal{M}(x)} F(x, y, z), \quad u(x)=\inf _{y \in \mathcal{M}(x)} w(x, y) \quad \text { and } \quad v=\inf _{x \in A} u(x) .
$$

Then

$$
w(x, y)= \begin{cases}-x_{2} y-\frac{1}{2} x_{1} x_{2} & \text { if } x_{2} \in\left[1, \frac{3}{2}[\right. \\ -\frac{3}{4} x_{1} & \text { if } x_{2}=\frac{3}{2} \\ -\frac{1}{2} x_{1} x_{2} & \text { if } \left.\left.x_{2} \in\right] \frac{3}{2}, 2\right]\end{cases}
$$

and

$$
u(x)= \begin{cases}-x_{2}-\frac{1}{2} x_{1} x_{2} & \text { if } x_{2} \in\left[1, \frac{3}{2}[\right. \\ -\frac{3}{4} x_{1} & \text { if } x_{2}=\frac{3}{2} \\ -\frac{1}{2} x_{1} x_{2} & \text { if } \left.\left.x_{2} \in\right] \frac{3}{2}, 2\right]\end{cases}
$$

Therefore, the infimal value of problem

$$
\min _{x \in A} \inf _{y \in \mathcal{M}(x)} \sup _{y \in \mathcal{M}(x)} F(x, y, z)
$$

is $v=-\frac{9}{4}$, but $(S)$ has no solution. Note that the multifunction $\mathcal{M}($.$) is not lower$ semicontinuous on $A$.

Before describing the steps of our investigation and giving its advantages, it is useful to give a brief survey on the study given in [1] considered in the finite dimensional case. For each $\epsilon>0$, the authors first present a regularized problem $\left(S_{\epsilon}\right)$ of $(S)$. The regularization is based on the use of $\epsilon$-approximate solutions of the lower level problem. Under mild assumptions, they show that $\left(S_{\epsilon}\right)$ admits solutions. Then, approximation results are obtained when the parameter of regularization $\epsilon$ goes to zero. This procedure of regularization has 
been inspired from the one given in [20] for weak bilevel problems. Our goal in this paper is to provide sufficient conditions under which the problem $(S)$ admits solutions. The study is considered in a sequential setting and based on a new regularization procedure and tools from variational convergence. This regularization uses strict $\epsilon$-solutions of the lower level problem and is inspired from those given in [1] and [20]. Under mild assumptions, we establish the existence of solutions for the regularized problem $\left(S_{\epsilon}\right)$. Note that for example in the case of real Hausdorff locally convex topological vector spaces, contrary to the regularization used in [1], this regularization allows us to avoid convexity assumptions. Then, via this regularization and under sufficient conditions, we show that the original bilevel problem $(S)$ has at least one solution. The obtained result can be applied to weak nonlinear bilevel problems in the finite and infinite dimensional cases by deleting the variable $y$. In particular, it extends and improves the result given in [3] for weak nonlinear bilevel programming problems. In fact, the result will be obtained under weaker assumptions without using convexity assumptions. Furthermore, our result gives a generalization from the linear finite dimensional case to the nonlinear infinite dimensional one of the result given in [26]. However, since the data are nonlinear, this generalization requires a new approach and other tools than those used in [26]. Note that one of the used sufficient conditions reduces the problem of existence of solutions for $(S)$ to a problem of the existence of a common solution of two parametric one-level optimization problems. Therefore, according to our study, we are led to investigate in one-level optimization instead of two-level optimization.

The outline of the paper is as follows. In Section 2, we recall and establish some basic results concerning the lower level problem. In section 3, we first present our regularization and prove the existence of solutions to the regularized problem under mild assumptions. Finally, we establish our main result on the existence of solutions to the original problem $(S)$.

\section{The Lower Level Problem: Basic Results}

In this section, we recall and establish some fundamental results concerning the lower level problem that we need in the sequel.

Definition 1 Let $Z$ be a Hausdorff topological space. We recall the following definitions

1) Let $C$ be a nonempty subset of $Z$. The set $\bar{C}^{\text {seq }}$ defined by

$$
\bar{C}^{\text {seq }}=\left\{a \in Z / \exists a_{n} \in C, a_{n} \rightarrow a, \text { as } n \rightarrow+\infty\right\}
$$

is called the sequential closure of $C$.

2) Let $\left(C_{n}\right)_{n \in \mathbb{N}}$ be a sequence of nonempty subsets of $Z$. The sets $\liminf _{n \rightarrow+\infty} C_{n}$ and $\limsup C_{n}$ are defined as follows $n \rightarrow+\infty$

i) $\liminf _{n \rightarrow+\infty} C_{n}=\left\{y \in Z / \exists y_{n} \rightarrow y\right.$, as $\left.n \rightarrow+\infty, y_{n} \in C_{n}, \forall n \in \mathbb{N}\right\}$,

ii) $\quad \limsup _{n \rightarrow+\infty} C_{n}=\left\{y \in Z / \exists y_{n_{k}} \rightarrow y\right.$, as $\left.k \rightarrow+\infty, y_{n_{k}} \in C_{n_{k}}, \forall k \in \mathbb{N}\right\}$.

Throughout the rest of the paper, the sets $A$ and $B$ will be equipped with the induced topologies of $X$ and $Y$ respectively. Moreover, we will always assume that $B$ is sequentially compact. 
Remark 1 In our case, the topological space $Y$ is first countable. So that, the topological closure and the sequential one coincide. In the sequel, we will work with the sequential closure because this notion will be associated with the sequential compactness of $B$.

We will use the following assumptions.

(2.1) The function $f$ is sequentially lower semicontinuous on the topological space $A \times B$,

(2.2) For any $(x, z) \in A \times B$, and any sequence $\left(x_{n}\right)$ converging to $x$ in $A$, there exists a sequence $\left(z_{n}\right)$ in $B$ such that

$$
\limsup _{n \rightarrow+\infty} f\left(x_{n}, z_{n}\right) \leq f(x, z),
$$

(2.3) For any $(x, z) \in A \times B$, and any sequence $\left(x_{n}\right)$ converging to $x$ in $A$, there exists a sequence $\left(z_{n}\right)$ converging to $z$ in $B$ such that

$$
\limsup _{n \rightarrow+\infty} f\left(x_{n}, z_{n}\right) \leq f(x, z) .
$$

For $x \in A$, set

$$
v(x)=\inf _{z \in B} f(x, z) .
$$

\section{Remark 2}

1) Assumption (2.2) is weaker than assumption (2.3). Note that assumptions (2.1)-(2.3) are weaker than the continuity of the function $f$ and are used in several papers; we cite for example [1-3, 6, 17, 19-21].

2) Assume that assumption (2.1) is satisfied. Then, since $B$ is sequentially compact, it follows that for any $x \in A$, the problem $\mathcal{P}(x)$ has at least one solution and $v(x)$ is a finite real number.

Let $x \in A$ such that $v(x)$ is a finite real number. For $\epsilon>0$, let $\mathcal{M}(\epsilon, x)$ and $\mathcal{M}^{s}(\epsilon, x)$ denote respectively the sets of $\epsilon$-approximate solutions and strict $\epsilon$-solutions of the lower level problem $\mathcal{P}(x)$, i.e.,

$$
\mathcal{M}(\epsilon, x)=\{z \in B / f(x, z) \leq v(x)+\epsilon\}
$$

and

$$
\mathcal{M}^{s}(\epsilon, x)=\{z \in B / f(x, z)<v(x)+\epsilon\} .
$$

Then, we have the following convergence results.

Proposition 1 Assume that assumptions (2.1) and (2.2) hold. Then, for any sequence $\left(x_{n}\right)$ converging to $x$ in $A$, we have

i) $\limsup \mathcal{M}\left(\epsilon, x_{n}\right) \subset \mathcal{M}(\epsilon, x), \forall \epsilon \geq 0$,

ii) $\lim \sup \mathcal{M}\left(\epsilon_{n}, x_{n}\right) \subset \mathcal{M}(x), \forall \epsilon_{n} \searrow 0^{+}$,

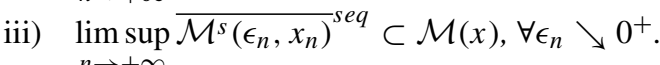

Proof For i) and ii) see [20]. We give the proof of iii). Since $f$ is sequentially lower semicontinuous on $A \times B$, then $\mathcal{M}\left(\epsilon_{n}, x_{n}\right)$ is sequentially closed on $B$. So that $\overline{\mathcal{M}^{s}\left(\epsilon_{n}, x_{n}\right)}{ }^{\text {seq }} \subset$ $\mathcal{M}\left(\epsilon_{n}, x_{n}\right)$, and the result follows by using ii). 
Proposition 2 Let $\epsilon>0$. Assume that assumptions (2.1) and (2.3) are satisfied. Then, for any sequence $\left(x_{n}\right)$ converging to $x$ in $A$, we have

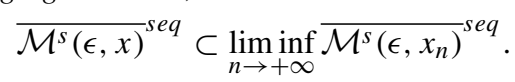

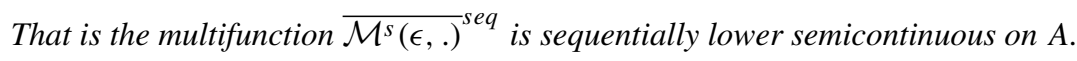

Proof From the sequential compactness of $B$ and assumptions (2.1) and (2.3), we have (see [20])

$$
\mathcal{M}^{s}(\epsilon, x) \subset \liminf _{n \rightarrow+\infty} \mathcal{M}^{s}\left(\epsilon, x_{n}\right)
$$

Then

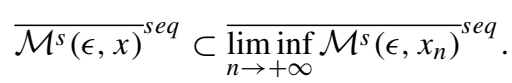

Since $Y$ is first countable, then, the set $\liminf _{n \rightarrow+\infty} \mathcal{M}^{s}\left(\epsilon, x_{n}\right)$ is closed. Therefore

$$
\begin{aligned}
{\overline{\mathcal{M}^{s}(\epsilon, x)}}^{s e q} & \subset \liminf _{n \rightarrow+\infty} \mathcal{M}^{s}\left(\epsilon, x_{n}\right) \\
& \subset \liminf _{n \rightarrow+\infty} \overline{\mathcal{M}^{s}\left(\epsilon, x_{n}\right)} \text { seq } .
\end{aligned}
$$

Remark 3 Since the set of strict $\epsilon$-solutions of the lower level problem is only defined for $\epsilon>0$, then, the case $\epsilon=0$ can not be considered in Proposition 2. However, for the case of $\epsilon$-approximate solutions, the sequential lower semicontinuity of the multifunction $\mathcal{M}($.) corresponding to the case where $\epsilon=0(\mathcal{M}()=.\mathcal{M}(0,)$.$) requires strong assumptions$ to be satisfied. This property plays a crucial role in the existence of solutions to weak and strong-weak bilevel problems.

\section{The First Level Problem: Existence of Solutions}

In this section, we first present our regularization and prove the existence of solutions to the regularized problem under mild assumptions. Then, under sufficient conditions, using the regularization and the notion of variational convergence, we establish the existence of solutions to problem $(S)$. We note that contrary to the study given in [1], no convexity properties are required for our investigation.

\subsection{The Regularized Problem : Existence of Solutions}

First, note that it is not difficult to show that the bilevel problem $(S)$ admits solutions under the lower semicontinuity of the multifunction $\mathcal{M}($.$) and additional mild assumptions.$ Unfortunately, as it is well-known in the literature, the property of lower semicontinuity of $\mathcal{M}($.$) is not satisfied in general. So that, the first step in our investigation is to substi-$ tute the multifunction $\mathcal{M}($.$) with \overline{\mathcal{M}}^{s(\epsilon, .)}$ seq which is lower semicontinuous under mild assumptions (Proposition 2). This substitution will be as follows.

For $\epsilon>0$, we consider the following regularized problem of $(S)$

$$
\left(S_{\epsilon}\right): \quad \min _{x \in A} \inf _{y \in \mathcal{M}(x)} \sup _{z \in \mathcal{M}^{s}(\epsilon, x)} F(x, y, z) .
$$


As mentioned above, such a regularization is new and is inspired from those given in [1] and [20]. Define the following marginal functions on $A \times B$ and $A$ respectively by

$$
w_{\epsilon}(x, y)=\sup _{z \in \mathcal{M}^{s}(\epsilon, x)} \operatorname{seq} F(x, y, z) \quad u_{\epsilon}(x)=\inf _{y \in \mathcal{M}(x)} w_{\epsilon}(x, y) .
$$

In order to prove the existence of solutions to problem $\left(S_{\epsilon}\right)$, we will show under sufficient conditions that the marginal function $u_{\epsilon}($.$) is sequentially lower semicontinuous on A$ sequentially compact.

The following assumptions will be used.

(3.1) The function $F$ is sequentially lower semicontinuous on $A \times B \times B$,

(3.2) The function $F$ is sequentially upper semicontinuous on $A \times B \times B$,

(3.3) For any $(x, y) \in A \times B$, the problems

$$
\max _{z \in B} F(x, y, z) \quad \text { and } \quad \min _{z \in B} f(x, z)
$$

admit a common solution.

For $(x, y) \in A \times B$, set $f_{x}()=.f(x,$.$) and F_{x, y}()=.F(x, y,$.$) .$

\section{Remark 4}

1) Assumption (3.3) generalizes the following one which was used in [3] for weak nonlinear bilevel programming problems, namely :

- For any $x \in A$, the following problems admit a common solution

$$
\max _{y \in B} f_{1}(x, y) \quad \text { and } \quad \min _{y \in B} f_{2}(x, y)
$$

where $f_{1}$ and $f_{2}$ are respectively the objective functions of the leader and the follower.

2) Assumption (3.3) is satisfied for example when $F$ and $f$ have the following decompositions

$$
F(x, y, z)=h(x, y)-\hat{h}(x, y) \mathcal{K}(x, z) \quad \text { and } \quad f(x, z)=g(x)+\hat{g}(x) \mathcal{K}(x, z)
$$

where $h, \hat{h}: X \times Y \rightarrow \mathbb{R}, g, \hat{g}: X \rightarrow \mathbb{R}$ and $\mathcal{K}: X \times Y \rightarrow \mathbb{R}$ are functions, with $\hat{h}(x, y)>0$ and $\hat{g}(x)>0, \forall(x, y) \in A \times B$.

3) Consider the case where $Y$ is a Banach space and assume that the following assumptions are satisfied

i) The set $B$ is convex,

ii) For every $x \in A$, the function $f_{x}$ (.) is convex and lower semicontinuous on $Y$,

iii) For every $(x, y) \in A \times B$, the function $F_{x, y}($.$) is concave and upper semi$ continuous on $Y$.

Then, assumption (3.3) can be written as

- For any $(x, y) \in A \times B$, there exists $z \in B$, such that

$$
0 \in\left[\partial f_{x}(z)+\mathcal{N}_{B}(z)\right] \cap\left[\partial\left(-F_{x, y}(z)\right)+\mathcal{N}_{B}(z)\right]
$$

where $\mathcal{N}_{B}(z)$ is the normal cone to $B$ at $z$ (see for example [9]).

We begin by the sequential lower semicontinuity of the marginal function $w_{\epsilon}(.,$.$) .$ 
Proposition 3 Let $\epsilon>0$. Assume that assumptions (2.1), (2.3) and (3.1) are satisfied. Then, the marginal function $w_{\epsilon}(.$, .) is sequentially lower semicontinuous on $A \times B$.

The result can be deduced from the results given in [17] in a general case. For the convenience of the reader, we give a short proof adapted to our case.

Proof Let $(x, y) \in A \times B$ and $\left(x_{n}, y_{n}\right)$ be a sequence converging to $(x, y)$ in $A \times B$. Let us show that

$$
\liminf _{n \rightarrow+\infty} w_{\epsilon}\left(x_{n}, y_{n}\right) \geq w_{\epsilon}(x, y) .
$$

Let $z \in{\overline{\mathcal{M}^{s}(\epsilon, x)}}^{s e q}$. From Proposition 2, there exists $z_{n} \rightarrow z$, as $n \rightarrow+\infty$ and $z_{n} \in{\overline{\mathcal{M}^{s}\left(\epsilon, x_{n}\right)}}^{\text {seq }}, \forall n \in \mathbb{N}$. Then

$$
w_{\epsilon}\left(x_{n}, y_{n}\right)=\sup _{t \in \mathcal{M}^{s}\left(\epsilon, x_{n}\right)} \operatorname{seq} F\left(x_{n}, y_{n}, t\right) \geq F\left(x_{n}, y_{n}, z_{n}\right) .
$$

It follows that

$$
\begin{aligned}
\liminf _{n \rightarrow+\infty} w_{\epsilon}\left(x_{n}, y_{n}\right) & \geq \liminf _{n \rightarrow+\infty} F\left(x_{n}, y_{n}, z_{n}\right) \\
& \geq F(x, y, z) .
\end{aligned}
$$

Since $z$ is arbitrary in ${\overline{\mathcal{M}^{s}(\epsilon, x)}}^{s e q}$, it follows that

$$
\liminf _{n \rightarrow+\infty} w_{\epsilon}\left(x_{n}, y_{n}\right) \geq \sup _{z \in \mathcal{M}^{s}(\epsilon, x)} \operatorname{seq} F(x, y, z)=w_{\epsilon}(x, y) .
$$

Hence, the marginal function $w_{\epsilon}(.,$.$) is lower semicontinuous on A \times B$.

The following result shows the sequential lower semicontinuity on $A$ of the marginal function $u_{\epsilon}($.$) .$

Proposition 4 Let $\epsilon>0$. Assume that assumptions (2.1), (2.3) and (3.1) are satisfied. Then, the marginal function $u_{\epsilon}($.$) is sequentially lower semicontinuous on A$.

Proof Let $x \in A$ and $\left(x_{n}\right)$ be a sequence converging to $x$ in $A$. Let us show that

$$
\liminf _{n \rightarrow+\infty} u_{\epsilon}\left(x_{n}\right) \geq u_{\epsilon}(x) .
$$

Assume the contrary. Then, there exists $\alpha \in \mathbb{R}$ such that

$$
\liminf _{n \rightarrow+\infty} u_{\epsilon}\left(x_{n}\right)<\alpha<u_{\epsilon}(x) .
$$

Set

$$
\liminf _{n \rightarrow+\infty} u_{\epsilon}\left(x_{n}\right)=\lim _{\substack{n \rightarrow+\infty \\ n \in \mathcal{N}}} u_{\epsilon}\left(x_{n}\right)
$$

where $\mathcal{N}$ is an infinite subset of $\mathbb{N}$. Then, there exists $n_{0} \in \mathcal{N}$, such that

$$
u_{\epsilon}\left(x_{n}\right)<\alpha \quad \forall n \geq n_{0}, n \in \mathcal{N} .
$$

So that, for all $n \geq n_{0}, n \in \mathcal{N}$, there exists $y_{n} \in \mathcal{M}\left(x_{n}\right)$ verifying

$$
w_{\epsilon}\left(x_{n}, y_{n}\right)<\alpha \text {. }
$$

Since $B$ is sequentially compact, then, there exists $\mathcal{N}^{\prime} \subset \mathcal{N}$ such that $y_{n} \rightarrow \bar{y}$, as $n \rightarrow+\infty, n \in \mathcal{N}^{\prime}$. Then, Proposition 1 implies that $\bar{y} \in \mathcal{M}(x)$. Therefore, by Proposition 3, we obtain

$$
w_{\epsilon}(x, \bar{y}) \leq \liminf _{n \rightarrow+\infty} w_{\epsilon}\left(x_{n}, y_{n}\right) \leq \alpha .
$$


So that

$$
u_{\epsilon}(x) \leq \alpha
$$

which gives a contradiction with the second strict inequality in (2).

Then, we obtain the following result on the existence of solutions to the regularized problem.

Theorem 1 Let $\epsilon>0$. Assume that assumptions (2.1), (2.3) and (3.1) are satisfied. If moreover, A is sequentially compact, then, the regularized problem $\left(S_{\epsilon}\right)$ has at least one solution.

Proof From Proposition 4 the marginal function $u_{\epsilon}($.$) is sequentially lower semicontinuous$ on $A$. Therefore, the result follows from the sequential compactness of $A$.

\subsection{Existence of Solutions to Problem $(S)$}

In this section, under sufficient conditions, we will establish the existence of solutions to problem $(S)$.

First, for $\epsilon>0$ and $x \in A$, consider the following auxiliary problem

$$
S_{\epsilon}(x): \quad \min _{y \in \mathcal{M}(x)} \sup _{z \in \mathcal{M}^{s}(\epsilon, x)} \operatorname{seq} F(x, y, z) .
$$

Then, we have the following result on the existence of solutions to problem $S_{\epsilon}(x)$.

Proposition 5 Let $\epsilon>0$ and $x \in$ A. Let assumptions (2.1), (2.3) and (3.1) hold. Then, the problem $S_{\epsilon}(x)$ has at least one solution.

Proof From Proposition 3 the marginal function $w_{\epsilon}(.,$.$) is sequentially lower semicontinu-$ ous on $A \times B$. So that, the marginal function $w_{\epsilon}(x,$.$) is sequentially lower semicontinuous$ on $B$. On the other hand, the set $\mathcal{M}(x)$ is sequentially closed in $B$ sequentially compact. Then, $\mathcal{M}(x)$ is sequentially compact and the result follows.

As mentioned in the introduction, the notion of variational convergence will play an important role in our investigation. So that, we recall some results related to this notion. Throughout the rest of the paper, for given problems of the form

$$
(\mathcal{P}): \quad \min _{x \in \widehat{A}} \hat{f}(x) \quad \text { and } \quad(\mathcal{Q}): \quad \max _{x \in \widehat{B}} \hat{g}(x)
$$

we shall denote by $\operatorname{Argmin} \mathcal{P}$ and $\operatorname{Argmax} \mathcal{Q}$ their solution sets respectively, where $\hat{f}, \hat{g}$ : $Z \rightarrow \mathbb{R}$ are functions, $Z$ is a Hausdorff topological space, $\widehat{A}$ and $\widehat{B}$ are nonempty subsets of $Z$.

Definition $2[8,12]$ Let $\varphi_{n}, \varphi: Z \rightarrow \overline{\mathbb{R}}, n \in \mathbb{N}$ be functions. We say that the sequence $\left(\varphi_{n}\right)$ converges variationally to $\varphi$ in $Z$ if the following properties are satisfied

i) For any $y \in Z$ and any sequence $\left(y_{n}\right)$ converging to $y$ in $Z$, we have

$$
\liminf _{n \rightarrow+\infty} \varphi_{n}\left(y_{n}\right) \geq \varphi(y) .
$$


ii) For any $y \in Z$, there exists a sequence $\left(y_{n}\right)$ in $Z$ such that

$$
\limsup _{n \rightarrow+\infty} \varphi_{n}\left(y_{n}\right) \leq \varphi(y) .
$$

Consider the problems

$$
\left(\mathcal{P}_{\varphi_{n}}\right): \min _{y \in Z} \varphi_{n}(y) \quad \text { and } \quad\left(\mathcal{P}_{\varphi}\right): \min _{y \in Z} \varphi(y) .
$$

Then, we have the following well-known important convergence result concerning the solution sets of problems $\left(\mathcal{P}_{\varphi_{n}}\right)$ and $\left(\mathcal{P}_{\varphi}\right)$.

Theorem 2 [8, 12] Assume that the sequence $\left(\varphi_{n}\right)$ converges variationally to $\varphi$ in $Z$. Then

i) $\limsup _{n \rightarrow+\infty} \operatorname{Argmin} \mathcal{P}_{\varphi_{n}} \subset \operatorname{Argmin} \mathcal{P}_{\varphi}$,

ii) $\limsup \epsilon_{n}-\operatorname{Argmin} \mathcal{P}_{\varphi_{n}} \subset \operatorname{Argmin} \mathcal{P}_{\varphi}, \forall \epsilon_{n} \searrow 0^{+}$, where $\epsilon_{n}-\operatorname{Argmin} \mathcal{P}_{\varphi_{n}}$ denotes the set of $\epsilon_{n}$-approximate solutions of problem $\left(\mathcal{P}_{\varphi_{n}}\right)$.

Lemma 1 Assume that assumptions (2.1), (2.2), (3.1) and (3.2) are satisfied. Let $(t, s) \in$ $A \times B$ and $\left(t_{n}, s_{n}\right)$ be a sequence converging to $(t, s)$ in $A \times B$. For $n \in \mathbb{N}$, let $z_{n}$ be a common solution of problems

$$
\max _{z \in B} F\left(t_{n}, s_{n}, z\right) \quad \text { and } \quad \min _{z \in B} f\left(t_{n}, z\right) .
$$

Then, any accumulation point of the sequence $\left(z_{n}\right)_{n \in \mathbb{N}}$ solves the problems

$$
\max _{z \in B} F(t, s, z) \quad \text { and } \quad \min _{z \in B} f(t, z) .
$$

Proof Let $\bar{z}$ be an accumulation point of the sequence $\left(z_{n}\right)_{n \in \mathbb{N}}$. Since $Y$ is first countable, then, there exists $\mathcal{N} \subset \mathbb{N}$ such that $z_{n} \rightarrow \bar{z}$ as $n \rightarrow+\infty, n \in \mathcal{N}$. Since $z_{n} \in \mathcal{M}\left(t_{n}\right)$, for $n \in \mathcal{N}$, it follows that $\bar{z} \in \lim \sup _{n \rightarrow+\infty} \mathcal{M}\left(t_{n}\right) \subset \mathcal{M}(t)$ (Proposition 1). That is $\bar{z}$ solves the problem

$$
\min _{z \in B} f(t, z) .
$$

Let us show that $\bar{z}$ solves the problem

$$
\max _{z \in B} F(t, s, z) .
$$

Assume that there exists $z^{*} \in B$ such that

$$
F(t, s, \bar{z})<F\left(t, s, z^{*}\right) .
$$

Then, the sequential continuity of $F$ implies that

$$
F\left(t_{n}, s_{n}, z_{n}\right)<F\left(t_{n}, s_{n}, z^{*}\right) \quad \text { for large } n \in \mathcal{N}
$$

which contradicts the optimality of $z_{n}$ for large $n \in \mathcal{N}$ to the problem

$$
\max _{z \in Y} F\left(t_{n}, s_{n}, z\right) \text {. }
$$

In the sequel, for a subset $\widehat{B}$ of $B, \psi_{\widehat{B}}($.$) denotes the indicator function of the set \widehat{B}$, i.e.,

$$
\psi_{\widehat{B}}(x)= \begin{cases}0 & \text { if } y \in \widehat{B} \\ +\infty & \text { if } y \notin \widehat{B} .\end{cases}
$$


Let $\epsilon_{n} \searrow 0^{+}$. Let $(t, s) \in A \times B$ and $\left(t_{n}, s_{n}\right)_{n}$ be a sequence converging to $(t, s)$ in $A \times B$ and consider the following auxiliary problems

$$
\mathcal{R}_{n}\left(t_{n}, s_{n}\right): \quad \max _{z \in \mathcal{M}^{s}\left(\epsilon_{n}, t_{n}\right)} \operatorname{seq} F\left(t_{n}, s_{n}, z\right)
$$

and

$$
\mathcal{R}(t, s): \quad \max _{z \in \mathcal{M}(t)} F(t, s, z) .
$$

Then, $\mathcal{R}_{n}\left(t_{n}, s_{n}\right)$ and $\mathcal{R}(t, s)$ are respectively equivalent to the following problems

$$
\min _{z \in B}\left\{-F\left(t_{n}, s_{n}, z\right)+\psi_{\overline{\mathcal{M}^{s}\left(\epsilon_{n}, t_{n}\right)}}^{\operatorname{seq}}(z)\right\}
$$

and

$$
\min _{z \in B}\left\{-F(t, s, z)+\psi_{\mathcal{M}(t)}(z)\right\}
$$

in the sense that they have the same solution sets and opposite optimal values. Define the functions $h$ and $h_{n}$ on $B$ by

$$
h_{n}(z)=-F\left(t_{n}, s_{n}, z\right)+\psi \overline{\mathcal{M}^{s}\left(\epsilon_{n}, t_{n}\right)} \operatorname{seq}(z)
$$

and

$$
h(z)=-F(t, s, z)+\psi_{\mathcal{M}(t)}(z) .
$$

Then, we have the following convergence result.

Proposition 6 Assume that assumptions (2.1), (2.2) and (3.1)-(3.3) are satisfied. Then, for any $(t, s) \in A \times B$ and any sequence $\left(t_{n}, s_{n}\right)_{n}$ converging to $(t, s)$ in $A \times B$, we have

$$
\limsup _{n \rightarrow+\infty} \operatorname{Argmax} \mathcal{R}_{n}\left(t_{n}, s_{n}\right) \subset \operatorname{Argmax} \mathcal{R}(t, s) .
$$

Proof We will show that the sequence $\left(h_{n}\right)$ converges variationally to $h$ in $B$.

1) Let $z \in B$ and $\left(z_{n}\right)$ be a sequence converging to $z$ in $B$. Let us show that

$$
\liminf _{n \rightarrow+\infty} h_{n}\left(z_{n}\right) \geq h(z) .
$$

We distinguish the following cases.

i) If $z \in \mathcal{M}(t)$, then, $h(z)=-F(t, s, z)$. On the other hand, assumptions (3.1) and (3.2) imply that

$$
\lim _{n \rightarrow+\infty} F\left(t_{n}, s_{n}, z_{n}\right)=F(t, s, z) .
$$

So that, for $\eta>0$, there exists $n_{0} \in \mathbb{N}$ such that

$$
\left|F\left(t_{n}, s_{n}, z_{n}\right)-F(t, s, z)\right|<\eta \quad \forall n \geq n_{0}, n \in \mathbb{N} .
$$

It follows that

$$
-F(t, s, z)-\eta<-F\left(t_{n}, s_{n}, z_{n}\right) \leq-F\left(t_{n}, s_{n}, z_{n}\right)+\psi_{\overline{\mathcal{M}^{s}\left(\epsilon_{n}, t_{n}\right)}}^{\operatorname{seq}}\left(z_{n}\right) .
$$

Hence

$$
h(z)-\eta<h_{n}\left(z_{n}\right) \quad \forall n \geq n_{0}, n \in \mathbb{N} .
$$

Then

$$
\liminf _{n \rightarrow+\infty} h_{n}\left(z_{n}\right) \geq h(z)-\eta .
$$

Since $\eta$ is arbitrary, we deduce that

$$
\liminf _{n \rightarrow+\infty} h_{n}\left(z_{n}\right) \geq h(z) .
$$


ii) If $z \notin \mathcal{M}(t)$, then, $h(z)=+\infty$. Moreover, $z_{n} \notin{\overline{\mathcal{M}^{s}\left(\epsilon_{n}, t_{n}\right)}}^{\text {seq }}$ for large $n \in \mathbb{N}$. Otherwise, there exists an infinite subset $\mathcal{N} \subset \mathbb{N}$ such that $z_{n} \in \overline{\mathcal{M}^{s}\left(\epsilon_{n}, t_{n}\right)}$ seq

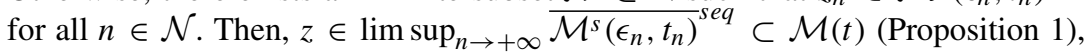
which gives a contradiction. We deduce that

$$
h_{n}\left(z_{n}\right)=+\infty \quad \text { for large } n \in \mathbb{N}
$$

and the result is obvious.

2) Let $\bar{z} \in B$. Let us show that there exists a sequence $\left(\bar{z}_{n}\right)$ in $B$ such that

$$
\limsup _{n \rightarrow+\infty} h_{n}\left(\bar{z}_{n}\right) \leq h(\bar{z}) .
$$

As above, we distinguish the following cases.

i) If $\bar{z} \notin \mathcal{M}(t)$, then, $h(\bar{z})=+\infty$, and the result is obvious.

ii) If $\bar{z} \in \mathcal{M}(t)$, then $h(\bar{z})=-F(t, s, \bar{z})$. According to assumption (3.3), for $n \in \mathbb{N}$, let $z_{n}^{*}$ be a common solution of problems

$$
\max _{z \in B} F\left(t_{n}, s_{n}, z\right) \quad \text { and } \quad \min _{z \in B} f\left(t_{n}, z\right) .
$$

We have $z_{n}^{*} \in \mathcal{M}\left(t_{n}\right) \subset{\overline{\mathcal{M}}\left(\epsilon_{n}, t_{n}\right)}^{s e q}$. Then, $h_{n}\left(z_{n}^{*}\right)=-F\left(t_{n}, s_{n}, z_{n}^{*}\right)$. Let $\mathcal{N}$ be an infinite subset of $\mathbb{N}$ such that

$$
\begin{aligned}
\limsup _{n \rightarrow+\infty} h_{n}\left(z_{n}^{*}\right) & =\limsup _{\substack{n \rightarrow+\infty\\
}}\left\{-F\left(t_{n}, s_{n}, z_{n}^{*}\right)\right\} \\
& =\lim _{\substack{n \rightarrow+\infty \\
n \in \mathcal{N}}}\left\{-F\left(t_{n}, s_{n}, z_{n}^{*}\right)\right\} .
\end{aligned}
$$

Using the sequential compactness of $B$, there exists $\mathcal{N}^{\prime} \subset \mathcal{N}$ such that $z_{n}^{*} \rightarrow z^{*}$ as $n \rightarrow+\infty, n \in \mathcal{N}^{\prime}$. That is $z^{*}$ is an accumulation point of the sequence $\left(z_{n}^{*}\right)_{n \in \mathbb{N}}$. According to Lemma $1, z^{*}$ solves the problems

$$
\max _{z \in B} F(t, s, z) \quad \text { and } \quad \min _{z \in B} f(t, z) .
$$

So that

$$
\begin{aligned}
\limsup _{n \rightarrow+\infty} h_{n}\left(z_{n}^{*}\right) & =\lim _{\substack{n \rightarrow+\infty \\
n \in \mathcal{N}}}\left\{-F\left(t_{n}, s_{n}, z_{n}^{*}\right)\right\} \\
& =\lim _{\substack{n \rightarrow+\infty \\
n \in \mathcal{N}^{\prime}}}\left\{-F\left(t_{n}, s_{n}, z_{n}^{*}\right)\right\} \\
& =-F\left(t, s, z^{*}\right) \\
& \leq-F(t, s, \bar{z})=h(\bar{z}) .
\end{aligned}
$$

We conclude that the sequence $\left(h_{n}\right)$ converges variationally to $h$. Then, using the result of Theorem 2, we obtain

$$
\limsup _{n \rightarrow+\infty} \operatorname{Argmax} \mathcal{R}_{n}\left(t_{n}, s_{n}\right) \subset \operatorname{Argmax} \mathcal{R}(t, s) .
$$

Let $\epsilon_{n} \searrow 0^{+}$and $t$ be an arbitrary point in $A$. Let us consider the following parametric minimization problems

$$
\left(\mathcal{Q}_{t, n}\right): \quad \min _{y \in \mathcal{M}(t)} \sup _{z \in \mathcal{M}^{s}\left(\epsilon_{n}, t\right)} \operatorname{seq} F(t, y, z)
$$


and

$$
\left(\mathcal{Q}_{t}\right): \quad \min _{y \in \mathcal{M}(t)} \sup _{z \in \mathcal{M}(t)} F(t, y, z)
$$

For $(x, y) \in A \times B$ and $n \in \mathbb{N}$, set

$$
w(x, y)=\sup _{z \in \mathcal{M}(x)} F(x, y, z)
$$

and

$$
\begin{aligned}
& w_{n}(x, y)=w_{\epsilon_{n}}(x, y) \\
& =\sup _{z \in \overline{\mathcal{M}}^{s}\left(\epsilon_{n}, x\right)} \operatorname{seq} F(x, y, z) .
\end{aligned}
$$

Proposition 7 Assume that assumptions (2.1), (2.2) and (3.1)-(3.3) are satisfied. For $n \in \mathbb{N}$, let $y_{t, n}$ be a solution of problem $\left(\mathcal{Q}_{t, n}\right)$ and $y_{t}$ be an accumulation point of the sequence $\left(y_{t, n}\right)_{n \in \mathbb{N}}$. Then, $y_{t}$ solves the problem $\left(\mathcal{Q}_{t}\right)$, i.e.,

$$
\limsup _{n \rightarrow+\infty} \operatorname{Argmin} \mathcal{Q}_{t, n} \subset \operatorname{Argmin} \mathcal{Q}_{t} .
$$

Proof Feasibility: We have $\left(y_{t, n}\right)_{n \in \mathbb{N}} \subset \mathcal{M}(t)$ which is sequentially closed. Then, $y_{t} \in$ $\mathcal{M}(t)$.

Optimality: Assume that there exists $y^{*} \in \mathcal{M}(t)$ such that

$$
w\left(t, y^{*}\right)=\sup _{z \in \mathcal{M}(t)} F\left(t, y^{*}, z\right)<w\left(t, y_{t}\right)
$$

So that

$$
F\left(t, y^{*}, z\right)<w\left(t, y_{t}\right) \quad \forall z \in \mathcal{M}(t) .
$$

There exists $\mathcal{N}_{1} \subset \mathbb{N}$ such that $y_{t, n} \rightarrow y_{t}$ as $n \rightarrow+\infty, n \in \mathcal{N}_{1}$. For $n \in \mathcal{N}_{1}$, let $z_{t, n}$ be a solution of the problem

$$
\mathcal{R}_{n}\left(t, y_{t, n}\right): \quad \max _{z \in \overline{\mathcal{M}}^{s}\left(\epsilon_{n}, t\right)} \operatorname{seq} F\left(t, y_{t, n}, z\right)
$$

i.e.,

$$
w_{n}\left(t, y_{t, n}\right)=F\left(t, y_{t, n}, z_{t, n}\right)
$$

(such a solution exists since the function $F\left(t, y_{t, n},.\right)$ is sequentially continuous and $\overline{\mathcal{M}}^{s}\left(\epsilon_{n}, t\right){ }^{s e q}$ is sequentially compact). From the sequential compactness of $B$, there exists an infinite subset $\mathcal{N}_{2}$ of $\mathcal{N}_{1}$ such that the sequence $\left(z_{t, n}\right)_{n \in \mathcal{N}_{2}}$ converges to $z_{t} \in B$. Then, Proposition 6 implies that

$$
z_{t} \in \limsup _{n \rightarrow+\infty} \operatorname{Argmax} \mathcal{R}_{n}\left(t, y_{t, n}\right) \subset \operatorname{Argmax} \mathcal{R}\left(t, y_{t}\right) .
$$

That is $z_{t}$ solves the problem

$$
\mathcal{R}\left(t, y_{t}\right): \quad \max _{z \in \mathcal{M}(t)} F\left(t, y_{t}, z\right) .
$$

So that

For $n \in \mathcal{N}_{2}$, let $z_{n}^{*} \in{\frac{\mathcal{M}}{\mathcal{M}^{s}\left(\epsilon_{n}, t\right)}}^{\text {seq }}$ such that

$$
w_{n}\left(t, y^{*}\right)=\sup _{z \in \overline{\mathcal{M}}^{s}\left(\epsilon_{n}, t\right)} \operatorname{seq} F\left(t, y^{*}, z\right)=F\left(t, y^{*}, z_{n}^{*}\right) .
$$


Let $\mathcal{N}_{3}$ be an infinite subset of $\mathcal{N}_{2}$ such that $\left(z_{n}^{*}\right)_{n \in \mathcal{N}_{3}}$ converges to $z^{*} \in B$. Proposition 1 implies that $z^{*} \in \mathcal{M}(t)$. Then, from (3), we have

$$
F\left(t, y^{*}, z^{*}\right)<w\left(t, y_{t}\right) .
$$

Using (5), we get

$$
F\left(t, y^{*}, z^{*}\right)<F\left(t, y_{t}, z_{t}\right) .
$$

So that, the sequential continuity of $F$ implies that

$$
F\left(t, y^{*}, z_{n}^{*}\right)<F\left(t, y_{t, n}, z_{t, n}\right) \quad \text { for large } n \in \mathcal{N}_{3} .
$$

Then, using respectively (6) and (4), we obtain

$$
w_{n}\left(t, y^{*}\right)<w_{n}\left(t, y_{t, n}\right) \quad \text { for large } n \in \mathcal{N}_{3}
$$

which contradicts the optimality of $y_{t, n}$ to the problem $\left(\mathcal{Q}_{t, n}\right)$ for large $n \in \mathcal{N}_{3}$.

Let $\epsilon_{n} \searrow 0^{+}$. For $x \in A$ and $n \in \mathbb{N}$, set

$$
u(x)=\inf _{y \in \mathcal{M}(x)} \sup _{z \in \mathcal{M}(x)} F(x, y, z) \quad \text { and } \quad u_{n}(x)=u_{\epsilon_{n}}(x) .
$$

Recall that

$$
\begin{aligned}
& u_{\epsilon_{n}}(x)=\inf _{y \in \mathcal{M}(x)} w_{\epsilon_{n}}(x, y)
\end{aligned}
$$

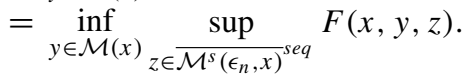

Now, we are able to state our main result on the existence of solutions to problem $(S)$.

Theorem 3 Let assumptions (2.1), (2.3) and (3.1)-(3.3) hold. If moreover, A is sequentially compact, then, the original strong-weak nonlinear bilevel problem $(S)$ has at least one solution.

Proof Let $\epsilon_{n} \searrow 0^{+}$. According to Theorem 1 , for $n \in \mathbb{N}$, let $\bar{x}_{n}$ be a solution of the regularized problem $\left(S_{\epsilon_{n}}\right)$. For $n \in \mathbb{N}$, let $\bar{y}_{n}$ be a solution of problem $S_{\epsilon_{n}}\left(\bar{x}_{n}\right)$ (Proposition 5). Since $A \times B$ is sequentially compact, then, there exists an infinite subset $\mathcal{N}$ of $\mathbb{N}$ such that the subsequence $\left(\bar{x}_{n}, \bar{y}_{n}\right)_{n \in \mathcal{N}}$ converges to $(\bar{x}, \bar{y}) \in A \times B$. Let us show that $\bar{x}$ solves the problem $(S)$.

Feasibility: We obviously have $\bar{x} \in A$.

Optimality: Assume that there exists $x^{*} \in A$ such that

$$
u\left(x^{*}\right)<u(\bar{x})
$$

i.e.,

$$
\inf _{y \in \mathcal{M}\left(x^{*}\right)} \sup _{z \in \mathcal{M}\left(x^{*}\right)} F\left(x^{*}, y, z\right)<\inf _{y \in \mathcal{M}(\bar{x})} \sup _{z \in \mathcal{M}(\bar{x})} F(\bar{x}, y, z) .
$$

Hence

$$
\inf _{y \in \mathcal{M}\left(x^{*}\right)} \sup _{z \in \mathcal{M}\left(x^{*}\right)} F\left(x^{*}, y, z\right)<\sup _{z \in \mathcal{M}(\bar{x})} F(\bar{x}, y, z) \quad \forall y \in \mathcal{M}(\bar{x}) .
$$

For $n \in \mathcal{N}$, we have $\bar{y}_{n}$ is a solution of problem

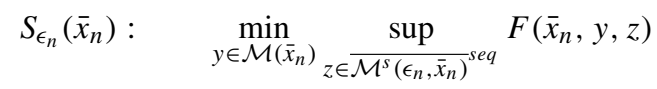


i.e.,

$$
\begin{aligned}
u_{n}\left(\bar{x}_{n}\right) & =\inf _{y \in \mathcal{M}\left(\bar{x}_{n}\right)} \sup _{z \in \mathcal{M}^{s}\left(\epsilon_{n}, \bar{x}_{n}\right)} \operatorname{seq} \\
& F\left(\bar{x}_{n}, y, z\right) \\
& =\sup _{z \in \overline{\mathcal{M}}^{s}\left(\epsilon_{n}, \bar{x}_{n}\right)}^{\operatorname{seq}} F\left(\bar{x}_{n}, \bar{y}_{n}, z\right) .
\end{aligned}
$$

Let $\bar{z}_{n} \in{\overline{\mathcal{M}^{s}\left(\epsilon_{n}, \bar{x}_{n}\right)}}^{\text {seq }}$ such that

$$
u_{n}\left(\bar{x}_{n}\right)=\sup _{z \in \overline{\mathcal{M}}^{s}\left(\epsilon_{n}, \bar{x}_{n}\right)} \operatorname{seq}\left(\bar{x}_{n}, \bar{y}_{n}, z\right)=F\left(\bar{x}_{n}, \bar{y}_{n}, \bar{z}_{n}\right)
$$

i.e., $\bar{z}_{n} \in \operatorname{Argmax} \mathcal{R}_{n}\left(\bar{x}_{n}, \bar{y}_{n}\right), n \in \mathcal{N}$ (such a point exists from the sequential compactness of the set $\overline{\mathcal{M}}^{s}\left(\epsilon_{n}, \bar{x}_{n}\right)$ seq and the sequential continuity of the function $F\left(\bar{x}_{n}, \bar{y}_{n},.\right)$ on $B$ ). Using again the sequential compactness of the set $B$, we deduce that there exists an infinite subset $\mathcal{N}_{1}$ of $\mathcal{N}$ such that the sequence $\left(\bar{z}_{n}\right)_{n \in \mathcal{N}_{1}}$ converges to $\bar{z}$. Proposition 6 implies that

$$
\bar{z} \in \limsup _{n \rightarrow+\infty} \operatorname{Argmax} \mathcal{R}_{n}\left(\bar{x}_{n}, \bar{y}_{n}\right) \subset \operatorname{Argmax} \mathcal{R}(\bar{x}, \bar{y}) .
$$

That is

$$
\sup _{z \in \mathcal{M}(\bar{x})} F(\bar{x}, \bar{y}, z)=F(\bar{x}, \bar{y}, \bar{z}) .
$$

On the other hand, for $n \in \mathcal{N}_{1}, \bar{y}_{n}$ solves the problem $S_{\epsilon_{n}}\left(\bar{x}_{n}\right)$. So that, $\bar{y}_{n} \in$ $\mathcal{M}\left(\bar{x}_{n}\right)$. Since $\left(\bar{y}_{n}\right)_{n \in \mathcal{N}_{1}}$ converges to $\bar{y}$, then it follows from Proposition 1 that $\bar{y} \in$ $\lim \sup _{n \rightarrow+\infty} \mathcal{M}\left(\bar{x}_{n}\right) \subset \mathcal{M}(\bar{x})$. Then, from (7), we have

$$
\inf _{y \in \mathcal{M}\left(x^{*}\right)} \sup _{z \in \mathcal{M}\left(x^{*}\right)} F\left(x^{*}, y, z\right)<\sup _{z \in \mathcal{M}(\bar{x})} F(\bar{x}, \bar{y}, z) .
$$

It follows from (9) that

$$
\inf _{y \in \mathcal{M}\left(x^{*}\right)} \sup _{z \in \mathcal{M}\left(x^{*}\right)} F\left(x^{*}, y, z\right)<F(\bar{x}, \bar{y}, \bar{z}) .
$$

For $n \in \mathcal{N}_{1}$, let $y_{x^{*}, n}$ be a solution of problem $\left(\mathcal{Q}_{x^{*}, n}\right)$ considered in Proposition 7 $\left(\left(\mathcal{Q}_{x^{*}, n}\right)\right.$ is obtained for $\left.t=x^{*}\right)$. That is

$$
\begin{aligned}
& u_{n}\left(x^{*}\right)=\inf _{y \in \mathcal{M}\left(x^{*}\right)} \sup _{z \in \mathcal{M}^{s}\left(\epsilon_{n}, x^{*}\right)} \operatorname{seq} F\left(x^{*}, y, z\right)
\end{aligned}
$$

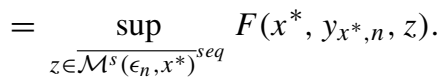

From the sequential compactness of $B$, there exists an infinite subset $\mathcal{N}_{2}$ of $\mathcal{N}_{1}$ such that $\left(y_{x^{*}, n}\right)_{n \in \mathcal{N}_{2}}$ converges to $y_{x^{*}}$. Therefore, $y_{x^{*}}$ solves the problem (see Proposition 7)

$$
\left(\mathcal{Q}_{x^{*}}\right): \quad \min _{y \in \mathcal{M}\left(x^{*}\right)} \sup _{z \in \mathcal{M}\left(x^{*}\right)} F\left(x^{*}, y, z\right) .
$$

That is

$$
\min _{y \in \mathcal{M}\left(x^{*}\right)} \sup _{z \in \mathcal{M}\left(x^{*}\right)} F\left(x^{*}, y, z\right)=\sup _{z \in \mathcal{M}\left(x^{*}\right)} F\left(x^{*}, y_{x^{*}}, z\right) .
$$

It follows from (10) that

$$
\sup _{z \in \mathcal{M}\left(x^{*}\right)} F\left(x^{*}, y_{x^{*}}, z\right)<F(\bar{x}, \bar{y}, \bar{z}) .
$$

In (11), for $n \in \mathcal{N}_{2}$, let $z_{n}^{*} \in{\overline{\mathcal{M}^{s}\left(\epsilon_{n}, x^{*}\right)}}^{\text {seq }}$ such that

$$
u_{n}\left(x^{*}\right)=F\left(x^{*}, y_{x^{*}, n}, z_{n}^{*}\right) .
$$


Using the sequential compactness of $B$, we can find $\mathcal{N}_{3} \subset \mathcal{N}_{2}$ such that $z_{n}^{*} \rightarrow z^{*}$ which belongs to $\mathcal{M}\left(x^{*}\right)$ according to Proposition 1. Since $z^{*} \in \mathcal{M}\left(x^{*}\right)$, then from (12), we get

$$
F\left(x^{*}, y_{x^{*}}, z^{*}\right)<F(\bar{x}, \bar{y}, \bar{z}) .
$$

Hence

$$
F\left(x^{*}, y_{x^{*}, n}, z_{n}^{*}\right)<F\left(\bar{x}_{n}, \bar{y}_{n}, \bar{z}_{n}\right) \quad \text { for large } n \in \mathcal{N}_{3} .
$$

Using respectively (13) and (8), we obtain

$$
u_{n}\left(x^{*}\right)<u_{n}\left(\bar{x}_{n}\right) \quad \text { for large } n \in \mathcal{N}_{3}
$$

which contradicts the optimality of $\bar{x}_{n}$ to the problem $\left(S_{\epsilon_{n}}\right)$, for large $n \in \mathcal{N}_{3}$.

Remark 5 Theorem 3 is also valid for weak nonlinear bilevel problems in the finite and infinite dimensional cases by deleting the variable $y$. Then, by application of this Theorem, we obtain under weaker assumptions without assuming convexity properties the result given in [3] for weak nonlinear bilevel programming problems. So that, our result constitutes an extension and an improvement of the result given in [3]. Moreover, it gives a generalization from the linear finite dimensional case to the nonlinear infinite dimensional one of the result given in [26]. However, according to our investigation, this generalization required a new approach and tools different from those used in [26]. Furthermore, by means of the property in assumption (3.3), we are led to investigate in one-level optimization instead of two-level optimization (see the remark below).

Remark 6 The property considered in assumption (3.3) concerns two parameterized problems in one-level optimization. In order to verify this property, we are led to verify if the solution sets of these two problems have a nonempty intersection. So that, by means of Theorem 3, the problem of the existence of solutions to $(S)$ is reduced principally to the problem of the existence of a common solution of two parameterized one-level optimization problems.

For illustration, let us consider the following example where all assumptions of Theorem 3 are satisfied.

Example 4 Let $X=\mathbb{R}^{2}, Y=\mathbb{R}, A=[1,2] \times[1,2]$ and $B=[1,2]$. Let $F$ and $f$ be the functions defined on $\mathbb{R}^{2} \times \mathbb{R} \times \mathbb{R}$ and $\mathbb{R}^{2} \times \mathbb{R}$ respectively by

$$
\begin{gathered}
F(x, y, z)=-\left(x_{1}+\frac{1}{2} x_{2}^{2}\right) y z^{2} \\
f(x, z)=\left(x_{1}-1\right) x_{2}^{3} z^{2}+\frac{1}{2}\left(1-x_{1}\right)^{2} x_{2}
\end{gathered}
$$

with $x=\left(x_{1}, x_{2}\right)$. It is easy to see that assumptions (2.1), (2.3), (3.1) and (3.2) are satisfied and $A$ and $B$ are compact. Let us verify assumption (3.3). For $(x, y) \in A \times B$, consider the problems

$$
\mathcal{P}(x): \quad \min _{z \in[1,2]} f(x, z)
$$

and

$$
Q(x, y): \quad \max _{z \in[1,2]} F(x, y, z)
$$


which admit respectively the following solution sets

$$
\mathcal{M}(x)= \begin{cases}{[1,2]} & \text { if } x_{1}=1 \\ \{1\} & \text { if } \left.\left.x_{1} \in\right] 1,2\right]\end{cases}
$$

and

$$
\widehat{\mathcal{M}}(x, y)=\{1\}
$$

Then, $z=1$ is a common solution to the problems $\mathcal{P}(x)$ and $Q(x, y)$. Hence, assumption (3.3) is satisfied. So that, according to Theorem 3 the solution set of the bilevel problem

$$
(S): \quad \min _{\substack{x_{i} \geq 1 \\ x_{i} \leq 2 \\ i=1,2}} \inf _{y \in \mathcal{M}(x)} \sup _{z \in \mathcal{M}(x)}\left\{-\left(x_{1}+\frac{1}{2} x_{2}^{2}\right) y z^{2}\right\}
$$

is nonempty.

The following example shows that assumption (3.3) is not necessary for the existence of solutions.

Example 5 Let $X=Y=\mathbb{R}, A=[2,3]$ and $B=[-5,5]$. Let $F$ and $f$ be the functions defined on $\mathbb{R} \times \mathbb{R} \times \mathbb{R}$ and $\mathbb{R} \times \mathbb{R}$ respectively by

$$
\begin{gathered}
F(x, y, z)=x-y-z \\
f(x, z)= \begin{cases}0 & \text { if } z^{2}-x^{2} \leq 0 \\
z^{2}-x^{2} & \text { if } z^{2}-x^{2}>0 .\end{cases}
\end{gathered}
$$

For $(x, y) \in A \times B$, consider the problems

$$
\mathcal{P}(x): \quad \min _{z \in[-5,5]} f(x, z)
$$

and

$$
Q(x, y): \max _{z \in[-5,5]} F(x, y, z) .
$$

So that, assumptions (2.1), (2.3), (3.1) and (3.2) are satisfied. Moreover, $A$ and $B$ are compact sets. With the same notations as above in Example 4, we have

$$
\mathcal{M}(x)=[-x, x] \quad \text { and } \quad \widehat{\mathcal{M}}(x, y)=\{-5\} .
$$

If follows that assumption (3.3) is not satisfied. On the other hand, we can easily check that $\bar{x}=2$ solves the problem

$$
(S): \quad \min _{x \in[2,3]} \inf _{y \in \mathcal{M}(x)} \sup _{z \in \mathcal{M}(x)}(x-y-z) .
$$

So that, assumption (3.3) is not necessary.

\section{Conclusion}

As we have mentioned, the study of the existence of solutions to strong-weak nonlinear bilevel problems is a difficult task. For illustration, the Example 3 presents a case where the objective functions are continuous and the decision variables range over compact sets, but the considered bilevel problem has no solutions. Then, our main result in Theorem 3 provides sufficient conditions ensuring the existence of solutions to such problems. For that, we have first given a regularization for problem $(S)$ which consists to substitute $\mathcal{M}($.) with a lower semicontinuous multifunction. Then, the main result is established via this 
regularization and the notion of variational convergence. The use of such a regularization allowed us to avoid convexity properties contrary to the one used in [1]. The obtained result can be applied to weak nonlinear bilevel problems in the finite and infinite dimensional cases by deleting the variable $y$. It is an extension and an improvement of the result given for weak nonlinear bilevel programming problems in [3]. It is also a generalization of the result given in [26] dealing with strong-weak bilevel problems. The generalization is from the linear finite dimensional case to the nonlinear infinite dimensional one. Another advantage of our result is that we are led to investigate in one-level optimization instead of two-level optimization. On the other hand, we note that the numerical study for such a class of problems is still in its infancy. The linear finite dimensional case was studied in [26], where numerical results are given. Another interesting subject is the numerical experiments of the nonlinear strong-weak bilevel problems. This is out of the scope of this paper and will be the subject of another paper.

\section{References}

1. Aboussoror, A., Loridan, P.: Strong-weak Stackelberg problems in finite dimensional spaces. Serdica Math. J. 21, 151-170 (1995)

2. Aboussoror, A., Loridan, P.: Existence of solutions to two-level optimization problems with nonunique lower-level solutions. J. Math. Anal. Appl. 254, 348-357 (2001)

3. Aboussoror, A.: Weak bilevel programming problems: existence of solutions. Adv. Math. Res. 1, 83-92 (2002)

4. Aboussoror, A., Mansouri, A.: Weak linear bilevel programming problems: existence of solutions via a penalty method. J. Math. Anal. Appl. 304, 399-408 (2005)

5. Aboussoror, A., Mansouri, A.: Existence of solutions to weak nonlinear bilevel problems via MinSup and D.C. problems. RAIRO Oper. Res. 42, 87-102 (2008)

6. Aboussoror, A., Adly, S., Jalby, V.: Weak nonlinear bilevel problems: existence of solutions via reverse convex and convex maximization problems. J. Ind. Manag. Optim. 7, 559-571 (2011)

7. Auslender, A., Crouzeix, J.-P.: Global regularity theorems. Math. Oper. Res. 13, 243-253 (1988)

8. Attouch, H.: Variational Convergence of Functions and Operators. Pitman, Boston (1984)

9. Azé, D.: Elements d'analyse convexe et variationnelle. Ellipses (1997)

10. Breton, M., Alj, A., Haurie, A.: Sequential Stackelberg equilibria in two-person games. J. Optim. Theory Appl. 59, 71-97 (1988)

11. Cao, D., Leung, L.C.: A partial cooperation model for non-unique linear two-level decision problems. Eur. J. Oper. Res. 140, 134-141 (2002)

12. Dontchev, A.L., Zolezzi, T.: Well-Posed Optimization Problems, Lecture Notes in Mathematics, vol. 1543. Springer Verlag, Berlin (1993)

13. Jia, S., Wan, Z., Feng, Y., Wang, G.: New partial cooperation model for bilevel programming problems. J. Syst. Eng. Electron. 22, 263-266 (2011)

14. Jia, S., Zheng, Y.: A new coordination model for ill-posed bilevel programming problem. Appl. Mech. Mater. 411-414, 1943-1947 (2013)

15. Jungers, M., Trélat, E., Abou-Kandil, H.: Min-max and min-min Stackelberg strategies with closed-loop information structure. J. Dyn. Control. Syst. 17, 387-425 (2011)

16. Leitmann, G.: On generalized Stackelberg strategies. J. Optim. Theory Appl. 26, 637-643 (1978)

17. Lignola, M.B., Morgan, J.: Semicontinuities of marginal functions in a sequential setting. Optimization 24, 241-252 (1994)

18. Liu, B., Wan, Z.: A partial cooperation model for bilevel programming problems with finite reactions follower. Oper. Res. Trans. 16, 84-92 (2012)

19. Loridan, P., Morgan, J.: New results on approximate solutions in two-level optimization. Optimization 20, 819-836 (1989)

20. Loridan, P., Morgan, J.: On strict $\epsilon$-solutions for a two-level optimization problem. In: Buhler, W., Feichtinger, G., Hartl, F., Radermacher, F.J., Stahly, P. (eds.) Operations Research Proceedings of the International Conference on Operations Research 90 in Vienna, pp. 165-172. Springer Verlag, Berlin (1992) 
21. Loridan, P., Morgan, J.: Weak via strong Stackelberg problem: new results. J. Glob. Optim. 8, 263-287 (1996)

22. Lucchetti, R., Mignanego, F., Pieri, G.: Existence theorems of equilibrium points in Stackelberg games with constraints. Optimization 18, 857-866 (1987)

23. Mallozzi, L., Morgan, J.: Hierarchical Systems with Weighted Reaction Set. Nonlinear Optimization and Applications, pp. 271-282. Plenum Press, New York (1996)

24. Mangasarian, O.L.: Nonlinear Programming. Mc Graw-Hill, New-York (1969)

25. Rockafellar, R.T.: Convex Analysis. Princeton University Press, Princeton (1970)

26. Zheng, Y., Wan, Z., Jia, S., Wang, G.: A new method for strong-weak linear bilevel programming problem. J. Ind. Manag. Optim. 11, 529-547 (2015) 